Traditionell schneidet die "Party in Power“ in Zwischenwahlen, wie es 2010 eine ist, schlecht(er) ab. Vom neuen Präsidenten Obama sowie den Fraktionsführungen im Kongress hängt es in den nächsten beiden Jahren wesentlich ab, wie erfolgreich die Arbeit von Exekutive und Legislative vom Wähler beurteilt wird. Von einer dauerhaften Demokratischen Mehrheit zu sprechen, wie es die Parteistrategen der Republikaner vor einigen Jahren angesichts eines Unified Government unter Präsident Bush getan haben, wäre zum gegenwärtigen Zeitpunkt verfrüht und vermutlich argumentativ nicht zu halten. ${ }^{51}$

51 Näheres dazu vgl. Hubert Silberhorn, Nachhaltige Dominanz? Präsident Bush, die Republikaner und ihr Masterplan, in: ZParl, 39. Jg. (2008), H. 1, S. 134 - 156; vgl. auch John Micklethwait I Adrian Wooldridge, The Right Nation. Conservative Power in America, S. 227 - 269, und Andy Barr, Dems talk of ,permanent progressive majority', in: Politico vom 7. November 2008, http:// www.politico.com/news/stories/1108/15407.html (Abruf am 15. Dezember 2008), sowie Robert G. Kaiser, Pollsters Debate America's Political Realignment, in: The Washington Post vom 23. November 2008; realistisch sieht der Vorsitzende des DCCC, Chris van Hollen, die Aussichten für das Wahljahr 2010: Statt einer dritten Demokratischen Welle gehe es darum, den gegenwärtigen Status Quo zu verteidigen, vgl. Andy Barr, Chris Hollen: No third Dem Wave in 2010, in: Politico vom 13. Februar 2009, http://www.politico.com/news/stories/0209/18828.html (Abruf am 7. April 2009).

\title{
Obamas Zeitenwende: Der Sieg allein ist nicht der Wechsel. Die US-Präsidentschaftswahl vom 4. November 2008
}

\author{
Bernhard Kornelius
}

Am 4. November 2008 wurde in den USA Geschichte geschrieben. Nach acht kontrovers diskutierten Jahren Bush-Administration, einer außergewöhnlich langen und stark polarisierenden Vorwahlphase sowie der teuersten Kampagne aller Zeiten vollzog sich unter den Augen der Weltöffentlichkeit und vor dem Hintergrund der weltweiten Wirtschafts- und Finanzkrise ein spektakulärer Wechsel. 44. Präsident der Vereinigten Staaten wurde mit Barack Obama zum ersten Mal ein farbiger Kandidat. Die Demokraten hatten - flankiert von Erfolgen in Senat und Repräsentantenhaus - nach zwei knappen Niederlagen in den Jahren $2000^{1}$ und $2004^{2}$ das Weiße Haus zurückerobert. Die Präsidentschaftswahl elektrisierte wie nur wenige andere Abstimmungen der letzten Jahre oder sogar Jahrzehnte: Schließlich ging es bei der Neubesetzung des weltweit wohl wichtigsten politischen Amtes um eine transnational signifikante Richtungsentscheidung.

Unabhängig von seiner politischen Tragweite und der hohen Symbolik war das Wahlergebnis per se weniger spektakulär: Der Erfolg des Demokraten Barack Obama über seinen

1 Vgl. Martin Thunert, Die US-amerikanischen Präsidentschaftswahlen vom 7. November 2000 kein Sieg Bushs, sondern eine Niederlage Gores, in: ZParl, 32. Jg. (2001), H. 3, S. 630 - 645.

2 Vgl. Michael Kolkmann, Die Präsidentschaftswahl in den USA vom 2. November 2004: Ein knapper Sieg Bushs, aber auch ein Mandat?, in: ZParl, 36. Jg. (2005), H. 2, S. 259 - 279. 
Republikanischen Gegenkandidaten John McCain war zwar ein deutlicher, aber kein überragender Sieg. Die Gründe für das Abschneiden beider Protagonisten sind vielschichtig und wie bei praktisch jeder demokratischen Wahl keinesfalls nur bestimmten soziodemographischen Gruppen, situativen Faktoren oder einem singulären Ereignis zuzuschreiben. Ein substanzielles Realignment in der US-amerikanischen Wählerschaft hat - das zeigt die nachfolgende Analyse - nicht stattgefunden. Vielmehr haben die politisch-administrative Ausgangslage und die ökonomischen Kontextbedingungen den in weiten Bevölkerungsteilen verbreiteten Wunsch nach einem grundsätzlichen Politikwechsel forciert zur Geltung gebracht. John McCain stand dabei trotz aller Distanzierungsversuche tendenziell für Kontinuität der Bush-Administration, Obama versprach hingegen den Wechsel zu einem anderen, zu einem neuen politischen Amerika.

\section{Vorwablen}

Vorausgegangen waren der General Election sowie dem Hauptwahlkampf die parteiinternen Vorwahlen in den einzelnen Bundesstaaten. ${ }^{3}$ Der Trend, wonach bereits amerikanische Vorwahlkämpfe besonders zeit- und ressourcenaufwendig sind, verstärkte sich nochmals massiv. Gleichzeitig lieferte eine vielerorts auf Rekordniveau angestiegene Wähler-Registrierung $^{4}$ zu diesem frühen Zeitpunkt klare Hinweise auf die immense Bedeutung, die die US-Bürger der anstehenden Präsidentschaftswahl zumessen sollten. Bei den Republikanern fiel die Vorentscheidung zugunsten von McCain infolge des Super-Tuesday Anfang Februar. Dabei war der 72-jährige Senator aus Arizona, Vietnam-Veteran und zweitältester Kandidat der Grand Old Party aller Zeiten, eigentlich nicht als Favorit gestartet. Bei den Demokraten endeten die Primaries und Caucuses ${ }^{5}$ als basisdemokratischer Selektionsprozess zur Kandidatenfindung ebenfalls mit einer Überraschung: Dass sich der 47-jährige Senator Barack Obama aus Illinois tatsächlich gegen die ehemalige First Lady und Senatorin für den Bundesstaat New York, Hillary Clinton, würde durchsetzen können, wurde jedoch erst mit Abschluss der sechsmonatigen Vorwahlphase im Juni 2008 zur Gewissheit.

\subsection{Ausgangslage der Republikaner}

Rund ein Jahr vor der Präsidentschaftswahl galt New Yorks ehemaliger Bürgermeister Rudy Giuliani bei den Republikanern als aussichtsreichster Bewerber. Auch Fred Thompson, ehemaliger Senator für den Südstaat Tennessee, lag in den landesweiten Umfragen unter „Like-

3 Alle Vorwahlergebnisse findet man unter http://politics.nytimes.com/election-guide/2008/results (Abruf am 1. März 2009).

4 Vgl. Katherine Q. Seelye, The Caucus. Records for Turnout, in: The New York Times vom 7. Februar 2008; Center for the Study of the American Electorate, 2008 Primary Turnout On Pace To Reach Record Highs, American University Media Relations vom 6. Februar 2008, http://www1. media.american.edu/pr.htm (Abruf am 3. März 2009).

5 Zur Unterscheidung der verschiedenen Vorwahltypen und Abstimmungsmodalitäten vgl. http:// www.thegreenpapers.com/Definitions.html (Abruf am 3. März 2009); Sven T. Siefken, Vorwahlen in Deutschland? Folgen der Kandidatenauswahl nach U.S.-Vorbild, in: ZParl, 33. Jg. (2002), H. 3, S. $531-550$. 
ly Republican Primary Voters“ zunächst gut im Rennen. Nach Erkenntnissen der meisten Meinungsforscher ${ }^{6}$ etwas stärker abgeschlagen - aber bei der oftmals unberechenbaren Eigendynamik der US-Vorwahlen keinesfalls chancenlos - präsentierten sich vor Beginn außerdem Mitt Romney, ehemaliger Gouverneur von Massachusetts, sowie Mike Huckabee, Baptistenprediger und Ex-Gouverneur von Arkansas. Ihm gelang es, trotz geringer Finanzmittel mit deutlichem Vorsprung vor Mitt Romney zu Jahresbeginn die erste Vorwahl in Iowa zu gewinnen. McCain folgte abgeschlagen auf Platz vier, Giuliani wurde lediglich Sechster. Gleichzeitig wurde ein signifikantes Defizit seiner riskanten Strategie sichtbar: Während der Iowa-Caucus traditionell den Auftakt des Vorwahlmarathons in den US-Bundesstaaten bildet und die meisten Kandidaten der Abstimmung im Mittleren Westen aufgrund seiner Symbolkraft ${ }^{7}$ viel Aufmerksamkeit widmen, konzentrierte sich Rudy Giuliani - programmatisch neokonservativ - fast ausschließlich auf Florida: Ein überzeugender Sieg im Sonnenscheinstaat knapp vier Wochen später, so das Kalkül auch anderer Bewerber ${ }^{8}$, würde der restlichen Kampagne den notwendigen Schub verleihen. In der Zwischenzeit hatte Giuliani aber auch in anderen Bundesstaaten empfindliche Niederlagen hinnehmen müssen und war letztendlich trotz massivem Mitteleinsatz Ende Januar in Florida ebenfalls gescheitert. New Yorks ehemaliger Bürgermeister gab umgehend seinen Rückzug bekannt ${ }^{9}$, nachdem mit Fred Thompson bereits kurz zuvor ein weiterer Kandidat wegen Erfolglosigkeit aufgegeben hatte.

Am Super-Tuesday, an dem die Republikanische Partei Anfang Februar in gleich 21 Bundesstaaten Vorwahlen abhielt, konzentrierte sich die Entscheidung faktisch noch auf drei Kandidaten. Diese repräsentierten gleichzeitig die drei programmatischen Hauptströmungen innerhalb der Grand Old Party: Erstens Mike Huckabee als Vertreter der „Social Conservatives“, der mit der Zielgruppe religiös-konservativer, primär weißer Amerikaner den Darwinismus kritisch betrachtete, Kreationismus und so genanntes Intelligent Design protegierte und sich gegen Abtreibung und Homo-Ehe aussprach; zweitens Mitt Romney, der die Vorwahlen in Michigan und Nevada gewinnen konnte und sich im Flügel der „Fiscal Conservatives“ für eine geringe Staatsquote und ein „small government"10 stark machte; und drittens John McCain, der in der Zwischenzeit in New Hampshire ein überraschendes Comeback geschafft hatte und anschließend das prestigeträchtige South Carolina ${ }^{11}$ sowie Florida gewann. Mit der Re-Vitalisierung der „Straight Talk“-Strategie ${ }^{12}$ stellte er als Vertre-

6 Umfrageergebnisse führender Institute vor und während des republikanischen Nominierungsprozesses, http://www.pollingreport.com/wh08rep.htm (Abruf am 1. März 2009).

7 Vgl. Christopher C. Hull, Grassroots Rules: How the Iowa Caucus Helps Elect American Presidents, Stanford 2007.

8 Neben Rudy Giuliani galt Florida auch für Mitt Romney und John McCain als eigentlicher Startpunkt der Kampagne. Vgl. Michael Levenson, Romney, McCain Look to Florida as Launch Pad, in: The Boston Globe vom 25. Januar 2008.

9 Vgl. Elisabeth Bumiller, Field Narrows as Giuliani and Edwards End Campaigns, in: The New York Times vom 30. Januar 2008.

10 Vgl. Michael Duffy / Karen Tumulty, Can the Economy Save Mitt Romney?, in: Time Magazine vom 17. Januar 2008.

11 Seit 1980 wurde immer derjenige Republikanische Bewerber zum Präsidentschaftskandidaten nominiert, der auch die Vorwahl in South Carolina gewonnen hatte.

12 Bei seiner erfolglosen Kandidatur im Jahr 2000 setzte John McCain auf einen ähnlichen Ansatz, vgl. Alison Mitchell, The 2000 Campaign: McCain Plans Strategy for Long Haul, in: The New York Times vom 21. Januar 2000. 
ter der „Foreign Affairs Conservatives“ transnationale Themen rund um Terrorismus und Irak ins Zentrum der Kampagne. Innenpolitisch vermied er eine zu starke Polarisierung und vertrat - innerhalb des Republikanischen Lagers - vergleichsweise liberale, partiell auch unbequeme Ansätze. Mit diesem Spagat war es McCain gelungen, nicht nur die Republikanische Kernwählerschaft anzusprechen, sondern bereits während der Vorwahlen auch unabhängige Wähler zu mobilisieren. Gleichzeitig hatte der Senator aus Arizona inzwischen die Unterstützung der ausgeschiedenen Kandidaten Giuliani und Thompson erhalten und fand unter anderem auch in Kaliforniens Gouverneur Arnold Schwarzenegger einen wichtigen Unterstützer, denn im bevölkerungsreichsten Bundesstaat waren die meisten Delegiertenstimmen zu holen.

Am Ende der großen Vorwahlschlacht des 5. Februar, die bei nie da gewesener Medienaufmerksamkeit schon fast den Charakter einer National Primary besaß, hatte McCain rund dreimal so viele Delegiertenstimmen wie Huckabee und viermal so viele Stimmen wie Romney gesammelt - zwar noch keine Mehrheit für die offizielle Nominierung, aber eine klare Bestätigung als Frontrunner der Republikaner für die noch ausstehenden Abstimmungen. Zwei Tage nach dem Super-Tuesday verkündete Romney seinen Ausstieg aus der Kampagne $^{13}$ und stellte sich nun ebenfalls hinter McCain. Huckabee blieb trotz klarer Niederlagen bei den Potomac Primaries in Maryland, Virginia und Washington D.C. zunächst noch im Rennen: Bei schwachen finanziellen Ressourcen, aber befeuert durch Zweifel am konservativen Profil seines Rivalen hielt Huckabee so lange seine Kandidatur aufrecht, bis McCain nach den Vorwahlen in Ohio, Rhode Island, Texas und Vermont am 4. März auch rechnerisch uneinholbar in Führung lag. Offiziell zum Präsidentschaftskandidaten gewählt wurde er dann auf dem Nominierungsparteitag der Republikaner Anfang September in Saint Paul, Minnesota.

\subsection{Ausgangslage der Demokraten}

Während die Entscheidung zugunsten John McCains vergleichsweise schnell gefallen war, lieferten sich Hillary Clinton und Barack Obama auf Seiten der Demokraten bis zum Abschluss der Vorwahlen eine erbitterte Auseinandersetzung. Weitere Kandidaten wie der Gouverneur von New Mexiko, Bill Richardson, oder die Senatoren Joseph Biden und Chris Dodd hatten bereits nach den ersten Abstimmungen zu Jahresbeginn aufgegeben. John Edwards verabschiedete sich Ende Januar aus dem Rennen: Gegen die übermächtigen Wahlkampfmaschinen der beiden Hauptkontrahenten war auch der Präsidentschaftskandidat des Jahres 2004 und spätere Kampfgefährte des damals nominierten Bewerbers John Kerry ohne jede Chance. ${ }^{14}$ Konträr zu den Protagonisten bei den Republikanern oder zu den eigenen Grabenkämpfen bei früheren Vorwahlen waren die programmatischen Differenzen zwischen Clinton und Obama eher gering; in den besonders relevanten Bereichen Finanzen, Gesundheit, Irak oder Umwelt waren kaum signifikante Unterschiede zu identifizieren.

13 Vgl. Dan Balz, Romney Out, McCain Looks Ahead, Apparent GOP Nominee Tries to Mend Fences With Conservatives, in: The Washington Post vom 8. Februar 2008.

14 Vgl. Adam Nagourney / JeffZeleny, The Final Two in a Multifront Battle, in: The New York Times vom 31. Januar 2008. 
Die eigentliche Überraschung war, dass es überhaupt zu einem lagerinternen Duell kam: Analog der politischen Stimmung in den meisten Umfragen ${ }^{15}$ galt die ehemalige First Lady für das Gros politischer Beobachter als klare Favoritin; in den eigenen Reihen gab es an einer Nominierung praktisch überhaupt keine Zweifel. Die Gründe ihres Scheiterns sind komplex: Einerseits offenbarte die Kampagne der Senatorin inhaltlich, organisatorisch und zuletzt auch finanziell Defizite. Andererseits wurden Obama und sein Team strategisch klar unterschätzt, obwohl Warnsignale wie Höhe, Herkunft und Struktur seiner Wahlkampfspenden bereits 2007 zu vernehmen waren; Details der organisatorischen Ausrichtung der lagerinternen Konkurrenz waren ebenfalls früh bekannt. Für jedermann sichtbar wurde das Potenzial des Senators aus Illinois - bis Ende 2006 auf nationaler Bühne weitgehend unbekannt - aber erst beim Vorwahlauftakt in Iowa: Dort, wo 95 Prozent der Bevölkerung Weiße sind und wo Clinton wie Edwards erhebliche Mittel investiert hatten, verbuchte Obama einen klaren Sieg und konstatierte einen "defining moment in history"16. Hillary Clinton wurde noch hinter John Edwards nur Dritte - sichtlich angeschlagen vollzog sie daraufhin einen taktischen Doppelwechsel: Nachdem sie zunächst gebetsmühlenartig unter dem Motto „Ready on Day One“ ihre jahrzehntelange Politikerfahrung in den Mittelpunkt gestellt hatte, versuchte sie sich mit einem „Change to Change“ nun vom Washingtoner Polit-Establishment abzusetzen. Gleichzeitig startete sie - unterstützt von Ehemann und Ex-Präsident Bill - forciertes „Negative Campaigning“. Doch gerade diejenigen Versuche, etwa mit Ausspielen der „Race Card“ Obamas Hautfarbe zu thematisieren oder ihn als „Establishment“-Kandidaten zu brandmarken, der nur der Gefühle wegen unterstützt würde $^{17}$, entpuppten sich als äußerst kontraproduktiv. Aggressive und persönliche Attacken auf den schwarzen Senator waren letztendlich mitverantwortlich dafür, dass Hillary Clinton in der Gunst potenzieller Demokratischer Wähler fiel; die New York Times unkte schon von einer „Billary Road to Republican Victory“18.

Hinzu kamen grundlegende strategische Fehler, von denen die folgenschwersten wohl die Konzentration von Geld und Kampagne auf den Super-Tuesday sowie auf den Gewinn vor allem der großen Bundesstaaten waren. Neben ihrem „Heimatstaat“ New York konnte die Senatorin am 5. Februar zwar acht weitere Bundesstaaten für sich entscheiden, darunter solch bedeutsame wie Massachusetts und Kalifornien. Mit ihrem Masterplan, nach diesem symbolträchtigen Vorwahltag unangefochtene Nummer Eins der Demokraten zu sein, war sie aber grandios gescheitert. Obama hatte nicht nur mehr Bundesstaaten gewonnen als seine Kontrahentin, sondern lag nach dem Super-Tuesday auch insgesamt knapp in Führung. Mit einigen guten zweiten Plätzen hatte er sich aufgrund der proportionalen Verteilung der direkt in den Vorwahlen ermittelten, so genannten Pledged Delegates weitere Stimmen gesichert und offenbarte so die eigentliche Basis seines Erfolges: Statt wie Clinton punktuell auf medienwirksame Erfolge in bevölkerungsreichen Staaten zu setzen, die - so das Kalkül - eine Dynamik im ganzen Land auslösen würden, war der vermeintliche Un-

15 Umfrageergebnisse führender Institute vor und während des Demokratischen Nominierungsprozesses mit Stand vom 31. Dezember 2008, http://www.pollingreport.com/wh08dem.htm.

16 Adam Nagourney, Obama Takes Iowa in a Big Turnout as Clinton Falters, in: The New York Times vom 4. Januar 2008.

17 Vgl. Associated Press, Bill Clinton Paints Obama as ,Establishment' Candidate, in: USA Today vom 15. Januar 2008.

18 Frank Rich, The Billary Road to Republican Victory, in: The New York Times vom 27. Januar 2008. 
derdog substanziell in der Fläche erfolgreich. Maßgeblich verantwortlich hierfür war eine in US-Wahlkämpfen bislang eher unkonventionelle Organisationsstruktur, die in einem engmaschigen Netz Wahlkampfteams und -büros in sämtlichen Bundesstaaten installiert hatte.

In ihrer Intensität bislang einmalig kamen neben den klassischen Instrumenten im „Air and Ground War" diverse elektronische Kommunikationsplattformen zum Tragen, die unter anderem den konstanten Fluss von Kleinspenden sicherstellten. ${ }^{19}$ Hillary Clinton war dagegen organisatorisch und vor allem finanziell nicht ausreichend auf eine Verlängerung der Auseinandersetzung über den Super-Tuesday hinaus vorbereitet, obwohl vergleichbare strategische Defizite anderer Bewerber bei zurückliegenden Präsidentschaftswahlen durchaus bekannt waren. Unmittelbar nach dem großen Vorwahltag musste Clinton überraschend einräumen, ihre Wahlkampfkasse mit fünf Millionen Dollar aus ihrem Privatvermögen auffüllen zu müssen. Um die Kampagne fortführen zu können, wurden in der Folgezeit weitere Privatzuschüsse und Kredite notwendig; Wahlkampfmanagerin Patti Solis Doyle wurde durch Clintons langjährige Vertraute Maggie Williams ersetzt. Obama konnte bei den Spenden hingegen Rekordeinnahmen verkünden - neben der absoluten Notwendigkeit einer stabilen finanziellen Basis für das Funktionieren der Kampagne ein in US-Wahlkämpfen auch psychologisch wichtiges Signal.

Die sich Mitte Februar anschließenden Vorwahlen in acht Bundesstaaten und Washington D.C. konnte Obama ausnahmslos für sich entscheiden. Vor allem aber konnte er jetzt mehr und mehr auch in denjenigen sozialen und demographischen Gruppen punkten, die aufgrund ihrer vermeintlich besonders hohen Reserviertheit gegenüber dem schwarzen Senator zunächst als möglicher Stolperstein auf dem Weg zur Präsidentschaft galten: Mit guten Ergebnissen unter anderem bei Frauen, Weißen oder Hispanics, vor allem aber auch in der weißen, formal weniger hoch gebildeten Mittelklasse der Blue Collar Workers wurden zunehmend substanzielle Wählerschichten aus dem Clinton-Lager gebunden. Der Rückzug Hillary Clintons ließ dennoch auf sich warten, vor allem, als diese Anfang März nach dem Gewinn von Ohio und einem Teilerfolg in Texas nochmals ihr Comeback zelebriert hatte. Trotz einiger weiterer Siege im April und Mai konnte sie Obama bei den Pledged Delegates nicht mehr überholen. Mehrfach versuchten Clinton und ihre Mitstreiter jetzt eine freizügige Umdeutung der Vorwahlergebnisse, indem sie auf eine vermeintliche Führung in der „Popular Vote“ verwiesen. ${ }^{20}$ Tatsächlich konnten diese Gesamtwählerstimmen zwar unterschiedlich interpretiert werden ${ }^{21}$; sie waren und sind im Nominierungsprozess eines demokratischen Präsidentschaftskandidaten aber letztendlich irrelevant, da - ähnlich der Wahl des Präsidenten im Wahlmännerkollegium - am Ende nur die Stimmen der Delegierten zählen. Erst nachdem auch immer mehr nicht gebundene Superdelegierte ${ }^{22}$ Barack Obama ihre Unterstützung zusagten - und so auch theoretisch die letzte Chance auf eine eigene Mehrheit geschwunden war -, beendete Clinton ihre eigenen Ambitionen ${ }^{23}$ : Nach einer

19 Vgl. Kapitel 2.4.

20 Vgl. Suzanne Smallay, What's the Magic Metric?, in: Newsweek vom 23. April 2008.

21 Vgl. Patrick Healy, As Time Runs Short, Clinton Claims Lead in Popular Vote, in: The New York Times vom 20. Mai 2008.

22 Übersicht aller Demokratischen „Pledged and Inpledged Delegates“, http://www.thegreenpapers. com/P08/D-PU.phtml (Abruf am 1. März 2009).

23 Vgl. Jonathan Weisman / Dan Balz, Clinton to Suspend Campaign, in: The Washington Post vom 5. Juni 2008. 
„langen und hart umkämpften Kampagne“24 verkündete sie im Anschluss an die allerletzten Primaries in South Dakota und Montana Anfang Juni in einer E-Mail an ihre Anhänger ihren Verzicht. Gleichzeitig appellierte sie an die bis dato tendenziell gespaltene Demokratische Partei, sich nun hinter Obama zu sammeln. Mit ihrem Votum für den einstigen Kontrahenten positionierte sich Clinton gleichzeitig für eine Aufgabe in einer kommenden Demokratischen US-Regierung - ein vollständiger Rückzug kam für die ehrgeizige Senatorin nicht infrage.

\section{Wahlkampf McCain vs. Obama}

Dass sich Barack Obama gegen Hillary Clinton überhaupt durchgesetzt hatte, galt vielfach als Sensation. Die eigentliche Hauptaufgabe stand dem 47 -jährigen Juristen aber noch bevor. Einerseits hatte die lange Auseinandersetzung um die Nominierung eindeutig zu seiner inhaltlichen Profilierung beigetragen. Der Gefahr, als „Verzauberer mit dem ewigen Lächeln (...) seine Faszination zu verlieren “25, begegnete er erfolgreich mit zunehmend sachlichen Auftritten. Andererseits hatte das innerparteiliche Duell den politischen Gegner wieder stärker ins Rennen gebracht: John McCain profitierte von dem anfänglich gespaltenen Demokratischen Lager. Zu Beginn der heißen Wahlkampfphase mit den Nominierungsparteitagen Ende August/Anfang September lagen die beiden Kontrahenten im so genannten Horse Race - der Frage der Demoskopen nach dem gewünschten Präsidenten ${ }^{26}$ - nahe beieinander. Signifikant absetzen konnte sich in den diversen Umfragen zu diesem Zeitpunkt keiner. Dabei waren die Unterschiede stark wie selten zuvor: Einem hochdekorierten Vietnam-Veteranen aus dem Washingtoner Polit-Establishment stand ein weitgehend unbekannter, wenngleich besonders charismatischer Afro-Amerikaner gegenüber - zwei Kandidaten, die darüber hinaus mit programmatisch zunächst differenzierten Ansätzen die USA zu führen gedachten und auch in ihrer Kampagne teils unterschiedliche Wege gingen. Wie in unzähligen Wahlkämpfen zuvor bildeten die drei Eckpfeiler „Message, Money and Mobilization“ hierfür das Grundkonstrukt.

\subsection{Kandidaten und Eigenschaften}

Allgegenwärtige Hauptbotschaft im US-Wahlkampf 2008 war die Forderung nach einem grundsätzlichen Wechsel. Losgelöst von spezifischen Themengebieten oder Politikansätzen wurde der vielzitierte „Change“ lagerübergreifend lanciert, entwickelte jedoch bei den Demokraten eine ungleich höhere Durchschlagskraft als bei den Republikanern. Mit der frühzeitigen Besetzung und einer konsequenten Einbindung als zentralem Kampagnenbegriff

24 Vollständiger Text der E-Mail unter http://blog.washingtonpost.com/44/2008/06/05/ clinton_ to_rally_party_behind.html (Abruf am 1. März 2009).

25 Martin Klingst, Die Reifeprüfung. Barack Obama gegen John McCain: Wie der Kampf um das Weiße Haus die Kandidaten verändert, in: Die Zeit vom 21. Mai 2008.

26 Das „Horse-Race Nationwide“ zwischen Obama und McCain erfreute sich gerade auch in den deutschen Medien höchster Beliebtheit, enthält aufgrund des Wahlsystems letztendlich aber keine ergebnisrelevanten Informationen. Vgl. Forschungsgruppe Wahlen, Daten zur Wahl der US-Präsidenten am 4. November 2008, Mannheim 2008, S. 5 f. 
traf Barack Obama nicht nur den Nerv einer ganzen Nation. Gleichzeitig war er der weitaus überzeugendere Vertreter einer Botschaft, die sich aufgrund der Ausgangslage als geradezu perfekt für die Mobilisierung erwies: Beim so genannten Track, der klassischen Frage der US-amerikanischen Meinungsforscher zur Lage der Nation, konstatierten 75 Prozent $^{27}$ - so viele Wähler wie noch nie -, dass sich die USA alles in allem in die falsche Richtung bewegten. 2004 lag dieser Wert noch bei 46 Prozent. ${ }^{28}$ Mitverantwortlich für diese Negativentwicklung war die amtierende Regierung und mit ihr an der Spitze George W. Bush. Dessen Zustimmungsrate, wiederum klassisches Messinstrument zur Amtsführung eines Präsidenten, war dramatisch von 53 Prozent vor der letzten Wahl auf 27 Prozent eingebrochen. In diesem Kontext wurde John McCain zum Verhängnis, dass fast die Hälfte aller Wähler von ihm die Fortsetzung der Politik des ungeliebten Amtsinhabers erwartete. Insbesondere in der Anhängerschaft des politischen Gegners glaubte nicht einmal jeder Zehnte, dass der Republikanische Bewerber das Land in eine andere Richtung führen würde. Trotz erheblicher Distanzierungs- und Absetzungsversuche war es McCain, der bereits bei seiner Kandidatur im Jahr 2000 mit der Selbstpositionierung eines non-konformen Einzelgängers gescheitert war, nicht gelungen, sich vollends von der „politischen Radioaktivität“ 29 des Weißen Hauses zu befreien.

\begin{tabular}{|c|c|c|c|}
\hline & alle & Obama-Wähler & McCain-Wähler \\
\hline „Er bringt den notwendigen Wechsel“ & 34 & 59 & 7 \\
\hline „Er vertritt meine Wertvorstellungen“ & 30 & 18 & 42 \\
\hline „Er hat die notwendige Erfahrung“" & 20 & 3 & 40 \\
\hline „Er kümmert sich um Leute wie mich“ & 12 & 17 & 6 \\
\hline
\end{tabular}

Dagegen war der wichtigste Grund für Obama zu stimmen, für 59 Prozent seiner Wähler die Überzeugung, dass er den notwendigen Wechsel tatsächlich herbeiführen werde (vgl. Tabelle 1). Vermeintlichen Defiziten in Sachen Erfahrung oder in der internationalen Politik, die nach Hillary Clinton dann auch McCain im Hauptwahlkampf als Argumente gegen eine Wahl Obamas in den Ring warf, stellte Obama Joe Biden gegenüber: Für das Amt des Vizepräsidenten schien der Vorsitzende des Außenpolitischen Ausschusses, Urgestein im Kongress und als Katholik mit spezifischem Rekrutierungspotenzial, die ideale Besetzung.

27 Die Quelle für alle nachfolgend aufgeführten Ergebnisse ist - wenn nicht ausdrücklich anders erwähnt - der Exit Poll vom 4. November 2008: Edison Media Research / Mitofsky International, United States General Election General Exit Poll 2008. Für die Befragung der Wähler unmittelbar nach der Wahl hatten wie schon 2004 die großen Networks abc, CBS, CNN, FOX News und NBC sowie AP das Meinungsforschungsinstitut Edison Media Research mit der Durchführung des Exit Polls beauftragt, auf dessen Basis in der Wahlnacht auch die Meldungen für die jeweiligen Bundesstaaten stattfanden. Für die US-Wahlberichterstattung der Forschungsgruppe Wahlen im ZDF während der Wahlnacht stand ebenfalls diese Datenbasis zu Verfügung.

28 Edison Media Research / Mitofsky International, United States General Exit Poll 2004, http:// www.exit-poll.net/ (Abruf am 17. April 2009).

29 So Michael Werz, Länderanalyse USA: Das Ende eines amerikanischen Jahrhunderts, in: Friedrich Ebert Stiftung (Hrsg.), Internationale Politikanalyse, Bonn 2009, S. 7. 
Tatsächlich hielten zwei Drittel der Amerikaner Biden auch für geeignet, im Notfall den Präsidenten zu ersetzen. Der Kandidatin der Gegenseite für den symbolträchtigen VizePosten hatten die meisten Wähler diese Qualifikation dagegen abgesprochen: Alaskas Gouverneurin Sarah Palin, die bei den Republikanern besonders das konservative Klientel binden sollte, erwies sich nach kurzzeitigem Zuspruch für die Kampagne McCains als kontraproduktiv: Ausgerechnet in den Politikbereichen, in denen die Republikaner Obama inhaltlich stellen wollten, wurden bei der eigenen Kandidatin für das Amt des Vizepräsidenten erhebliche Mängel sichtbar.

Für die Wähler waren die Unterschiede bei der im Vorfeld vieldiskutierten Kandidateneigenschaft Erfahrung letztendlich gering: So sprachen 48 Prozent Obama, aber auch 40 Prozent $M c$ Cain diese Eigenschaft ab; 49 Prozent sahen beim Demokraten und 59 Prozent beim Republikaner ausreichend Erfahrung. Was das notwendige Urteilsvermögen im Präsidentenamt betrifft, lag Obama sogar vor McCain: 59 Prozent aller Wähler attestierten ihm entsprechende Qualitäten, 49 Prozent waren es mit Blick auf seinen Gegner. Andere Kandidateneigenschaften, die ebenfalls mehr oder weniger stark für das Negative Campaigning instrumentalisiert wurden, kamen fast überhaupt nicht zum Tragen: Für 90 Prozent aller Wähler war die Hautfarbe der Kandidaten bei der Wahl kein relevanter Faktor, und zwar ohne jeden Unterschied in den Parteianhängerschaften. Etwas stärkere Differenzen gab es beim Alter der Kandidaten: Für sieben Prozent der $M c$ Cain-Wähler und für 23 Prozent der Obama-Wähler hatte das Alter - die beiden Protagonisten trennen 25 Jahre - hohes Gewicht bei der Entscheidung. Umgekehrt spielte aber auch dieser im Vorfeld der Wahl intensiv diskutierte Faktor für 84 Prozent keine Rolle. Die persönlichen Eigenschaften beziehungsweise Führungsqualitäten waren alles in allem ohnehin nur einer Minderheit von 39 Prozent der Wähler wichtiger als die politischen Standpunkte der Kandidaten, die für 59 Prozent der Amerikaner am 4. November 2008 das höhere Gewicht besaßen.

\subsection{Themen und Positionen}

Vor dem Hintergrund der aufziehenden Finanz- und Bankenkrise war die Präsidentschaftswahl eindeutig vom konjunkturellen Abschwung überlagert: 63 Prozent aller Wähler (65 Prozent der Obama-, aber auch 60 Prozent der McCain-Anhänger) benannten die Wirtschaftslage als wichtigstes Problem der USA (vgl. Tabelle 2). Nachdem noch im Jahr 2004 47 Prozent der Befragten die ökonomische Gesamtkonstitution des Landes positiv und 52 Prozent ${ }^{30}$ negativ beurteilt hatten, konstatierten jetzt 93 Prozent eine überwiegend schlechte und gerade noch sieben Prozent eine eher gute Wirtschaftslage - eine dramatische Entwicklung. Dass kein Präsident diese Probleme über Nacht würde lösen können, war den meisten Amerikanern offensichtlich bewusst: Für die kurz- und mittelfristige Konjunkturentwicklung waren 85 Prozent aller Befragten pessimistisch, 81 Prozent befürchteten außerdem für sich persönlich und ihre Familien im kommenden Jahr negative Konsequenzen. Tatsächlich prognostizierte die amerikanische Notenbank schon vor der Wahl ein nur noch marginales Wachstum für 2009; die Leitzinsen wurden im Wahljahr um über drei Punkte gesenkt, und auf dem Arbeitsmarkt standen Massenentlassungen an.

30 Edison Media Research / Mitofsky International, a.a.O. (Fn. 28). 
Um die Folgen der Wirtschafts- und Finanzkrise für die Bürger möglichst gering zu halten, setzten beide Kandidaten im Wahlkampf - früher oder später - auf staatliche Konjunkturprogramme, direkte Entlastungen der Bürger sowie eine strengere Aufsicht der Finanzmärkte. ${ }^{31} \mathrm{Im}$ Detail waren die Ansätze zwar unterschiedlich ${ }^{32}$, doch während Obama sich frühzeitig für neue Kontrollgremien und restriktivere Regulierungsvorgaben stark machte, war McCain in diesem Punkt auch wegen parteiinterner Differenzen zunächst sehr zögerlich. Für das 700 Milliarden US-Dollar teure Rettungspaket der Regierung stimmten später beide Kandidaten, obwohl ausgerechnet beim Thema Staatsinterventionismus die wohl markanteste Trennlinie durch Amerikas Wählerschaft ging: Während bei den Demokraten eine klare Mehrheit von 72 Prozent zukünftig für mehr staatliche Eingriffe plädierte, vertrauten 69 Prozent der Republikanischen Anhänger in diesem Kontext eher auf die Selbstregulierungsfähigkeiten der Märkte. Etwa zu dem Zeitpunkt, als sich McCain - ehemals großer Verfechter von Marktfreiheit und Deregulierung - klar für mehr Marktaufsicht ausgesprochen und mit seinem ordnungspolitischen Kurswechsel inhaltlich von seiner Basis entfernt hatte, war Obama bei den landesweiten Umfragen erstmals sichtbar in Führung gegangen und verlor diese bis zur Wahl am 4. November 2008 auch nicht mehr.

\begin{tabular}{|c|c|c|c|}
\hline & alle & Obama-Wähler & McCain-Wähler \\
\hline Wirtschaftslage & 63 & 65 & 60 \\
\hline Krieg im Irak & 10 & 11 & 8 \\
\hline Terrorismus & 9 & 2 & 16 \\
\hline Gesundheitsvorsorge & 9 & 13 & 5 \\
\hline Energiepolitik & 7 & 7 & 7 \\
\hline
\end{tabular}

Die Agenda wurde zunehmend von Finanzkrise und ökonomischen Problemen bestimmt. Die Politikfelder Gesundheitsvorsorge und Energiepolitik, innenpolitisch heftig umstritten, waren angesichts der negativen Wirtschaftsentwicklung am Wahltag in den Hintergrund gerückt. Auch das Thema Irak, gut ein Jahr vor der eigentlichen Wahl noch von zentraler Bedeutung, war am 4. November mit zehn Prozent der Nennungen nur noch schwach präsent. Dennoch konzentrierten sich beide Kandidaten mit der in US-amerikanischen Wahlkämpfen gewohnten Intensität auch auf klassische außenpolitische Themenfelder: McCain, der so innenpolitische Defizite zu kompensieren, den vermeintlich unerfahrenen Kontrahenten zu diskreditieren sowie die eigene Klientel aufgrund mutmaßlicher Republikanischer Kompetenzvorteile zu mobilisieren versuchte; und Obama, der bei den internationalen Beziehungen eine signifikante Neujustierung versprach. Die besten Argumente hierfür und eine hervorragende Angriffsfläche gleichermaßen bot ausgerechnet der politische Gegner beziehungsweise die miserable außenpolitische Gesamtbilanz der Bush-Administration. Unter anderem waren beim Thema Irak die Mehrheiten in der Bevölkerung gekippt:

31 Vgl. Elizabeth Holmes / Nick Timiraos, McCain, Obama Confront the Market, in: The Wall Street Journal vom 16. September 2008.

32 Positionen Obamas zur Finanz- und Wirtschaftskrise, http://www.barackobama.com/2008/09/16/ remarks_of_senator_barack_obam_113.php); Positionen McCains, http://www.ontheissues.org/ 2008/john_mccain_budget_+_economy.htm (Abruf am 2. April 2009). 
Nach 51 Prozent im Jahr 2004 standen jetzt noch 36 Prozent der Befragten hinter dem Einsatz, und zwar höchst differenziert mit 68 Prozent der Republikanischen und lediglich neun Prozent der Demokratischen Anhänger. Während $M c$ Cain auf ein längerfristiges Engagement der US-Truppen setzte, forderte Obama zunächst einen Rückzug binnen 16 Monaten und dafür eine wesentlich stärkere Präsenz in Afghanistan. Dabei wurde McCain auch Opfer seines eigenes Erfolges, denn er hatte schon früh einen Strategiewechsel des US-Einsatzes am Golf, insbesondere eine massive Truppenaufstockung gefordert, die - partiell umgesetzt - inzwischen zur Stabilisierung der Lage vor Ort und somit zum Bedeutungsverlust des Themas bei den heimischen Wählern geführt hatte. Weit mehr Polarisierung gab es in puncto Iran, wo Obama ohne Vorbedingungen für bilaterale Verhandlungen, McCain dagegen für eine stärkere Isolierung Teherans eintrat. Grundsätzlich verfolgten jedoch beide Kandidaten eine klare Abkehr vom Unilateralismus der ersten Amtszeit George W. Bushs und hatten sich zum Ende des Wahlkampfes in vielen Bereichen inhaltlich angenähert. Übergeordnetes außenpolitisches Ziel war hier wie dort auch der Reputationsrückgewinn einer multilateral wieder stärker zu integrierenden Führungsnation, nicht zuletzt durch effektive Reaktivierung der transatlantischen Partnerschaft mit den Europäern. ${ }^{33}$

\subsection{Kampagne und Mobilisierung}

Zur Rekrutierung und Mobilisierung potenzieller Wähler kombinierten beide Lager bewährte Ansätze mit neuen beziehungsweise weiterentwickelten Wahlkampfinstrumenten. Neben unzähligen persönlichen Auftritten beider Kandidaten im ganzen Land, dem Einsatz der Parteibasis sowie einer hohen Präsenz in Radio und Fernsehen war das Internet ein elementarer Baustein beider Kampagnen. Natürlich ist der Einsatz modernster Kommunikationsmittel bei US-Wahlen kein neues Phänomen. Modellcharakter hatten etwa die ersten Fernsehdebatten und Radioansprachen oder - bereits im vorletzten Jahrhundert - die gezielte Einbindung der Presse. Die Nutzung des Internets in den Wahlkämpfen US-amerikanischer Präsidentschaftsbewerber ist so die konsequente Fortschreibung dieser Tradition, wobei die Kampagne Obamas für das 21. Jahrhundert neue Standards setzte: „By exploiting the potential of the internet as a tool for organizing, mobilizing, and fundraising, he ran the first true campaign of the 21 st century. " 34 Zentraler Unterschied zu bisherigen Online-Kampagnen war eine erhebliche Aufwertung des Instruments vom eindimensionalen Distributionskanal hin zur interaktiven Kommunikationsplattform. Über Portale wie YouTube, FaceBook oder MySpace und natürlich über die eigene Domain my.barackaobama.com wurden die Nutzer nicht nur gezielt umworben und angesprochen, sondern gleichzeitig als pro-aktives Element in die Kampagne eingebunden. Neben dem Spenden von Geld, der Bewertung bestimmter Internetseiten zwecks verbesserten Rankings in den Suchmaschinen oder dem Verfassen eigener Blogeinträge förderten diese Portale vor allem die Ausbildung diverser sozialer Netzwerke. Diese dienten wiederum zur Weiterverbreitung

33 Vgl. James P. Rubkin, Building a New Atlantic Alliance. Restoring America’s Partnership with Europe, in: Foreign Affairs, 87. Jg. (2008), H. 7/8, S. 99 - 110.

34 So William A. Galston, Obama Runs First True Campaign of 21st Century, http://www.brookings.edu/opinions/2008/0604_nominee_galston.aspx (Abruf am 30. März 2009). 
politischer Botschaften, der Organisation von Wahlkampfveranstaltungen und somit zur Rekrutierung neuer Anhänger. Durch eine verstärkte Bindung an einen Kandidaten ${ }^{35}$ besaß das politische Web 2.0 eine integrative Funktion; gleichzeitig gaben die Unterstützernetzwerke den Strategen eine stets hochaktuelle Rückmeldung der potenziellen Basis: Erwartungen, Wünsche oder Sorgen des Wählers konnten schnell und präzise identifiziert sowie anschließend höchst individuell bedient werden. Als quasi-eigenständige Bewegung waren die entsprechenden Plattformen zudem ein äußerst kosten- und ressourcenschonendes Instrument, was aufgrund seiner dezentralen Struktur jedoch auch zu Kontrollverlust und ungewünschter Eigendynamik führen kann sowie neue Möglichkeiten des Negative Campaigning eröffnet. ${ }^{36}$

Vor der US-Wahl fand das Internet als Werkzeug politischer Kampagnenführung gerade auch in den deutschen Medien große Beachtung. Dass es aber maßgeblich für den Wahlausgang gewesen sei, oder dass Obama gar die Wahl via Web 2.0 gewonnen habe, ist wenigstens eine "grobe Vereinfachung “37. Vielmehr wurden die entsprechenden Optionen als Ergänzung herkömmlicher Instrumente äußerst konsequent in seine Kampagne eingebunden und auch aufgrund struktureller Vorteile wesentlich effektiver umgesetzt als beim politischen Gegner. Selbstverständlich bediente sich McCain, bei seiner Kandidatur 2000 noch „Master of Online Technology“38, ebenfalls der zeitgemäßen Möglichkeiten. Konzeptionelle Schwächen, aber vor allem die Sozialstruktur der Republikanischen Anhängerschaft mit einer tendenziell geringeren Internetaffinität führten in diesem Lager zu einer weniger intensiven Nutzung des Mediums. Insgesamt 33 Prozent aller US-Amerikaner - 24 Prozent der Republikanischen Anhänger, aber 34 Prozent der Anhänger der Demokraten sowie 38 Prozent der Wahlberechtigten ohne Parteibindung - benannten das Internet als Informationsquelle im Wahlkampf. ${ }^{39}$ Daneben informierten sich 29 Prozent der US-Bürger über die Printmedien, 21 Prozent über das Radio und eine sehr deutliche Mehrheit von 72 Prozent über das Fernsehen. Während sich die Nutzungsintensität des Internets verglichen zu 2004 verdreifacht hat, sind die klassischen Formate in ihrer Reichweite weitgehend stabil. Dies wiederum zeigt einerseits, dass das Internet trotz seines hohen Bedeutungszuwachses ganz offensichtlich mehr als Ergänzung denn Alternative genutzt wird. Andererseits verdeutlichen allein die Relationen, dass das Fernsehen auch 2008 unangefochten die Hauptrolle spielte, wenn es um politische Massenkommunikation während der Präsidentschaftskampagnen geht.

35 Vgl. Lee Rainie / Aaron Smith, The Internet and the 2008 Election, in: Pew Research Center Publications vom 15. Juni 2008.

36 Vgl. Stefan Schmidt, Die Bedeutung des Internets im US-Präsidentschaftswahlkampf 2008, in: Deutscher Bundestag (Hrsg.), Wissenschaftliche Dienste FB WD 1, 264/08, Berlin 2009, S. 9 f.

37 So Garrett M. Graff, Per SMS ins Weiße Haus - Obama und die Neuen Medien, in: Friedrich Ebert Stiftung (Hrsg.), Fokus Amerika Nr. 1/2009, S. 2.

38 So Garrett M. Graff, McCain and the Internet: Why it Matters, http://www.washingtonian.com/ blogarticles/people/capitalcomment/8815.html (Abruf am 1. April 2009).

39 Mehrfachnennung möglich; Pew Research Center, Internet Now Major Source of Campaign News, in: Pew Research Center Publications vom 31. Oktober 2008. 


\subsection{Finanzen}

Völlig neue Dimensionen wurden bei den Wahlkampffinanzen erreicht. Traditionell bildet das den Kandidaten zur Verfügung stehende Spendenvolumen das Rückgrat der langwierigen und extrem teuren amerikanischen Wahlkämpfe. ${ }^{40}$ Letztendlich war es ein mitentscheidender Faktor für den späteren Erfolg des 44. US-Präsidenten: „The story of Obama's success is very much a story about money. " ${ }^{11}$ Schon bei der letzten Präsidentschaftswahl waren die für Vorwahlen, Nominierungsparteitage und Hauptwahlkampf eingesetzten Finanzmittel aller Kandidaten im Vergleich zum Jahr 2000 um 56 Prozent auf gut eine Milliarde USDollar hochgeschnellt. 2008 lag die vergleichbare Gesamtsumme bei spektakulären 1,673 Milliarden US-Dollar. ${ }^{42}$ Obama konnte mit fast 745 Millionen US-Dollar ein gut doppelt so hohes Budget einsetzen wie sein Hauptkontrahent McCain, dem Spenden in Höhe von insgesamt 347 Millionen US-Dollar zur Verfügung standen und der seine Ressourcen somit ungleich sparsamer einsetzen musste.

Neben dem Volumen gab es eine bemerkenswerte Entwicklung bei Herkunft und Struktur der Spenden, vor allem aber auch bei der externen Kommunikation dieses so wichtigen Themas: Während sich eine relative Mehrheit der Gelder, die an Obama flossen, aus so genannten Kleinspenden von bis zu 200 US-Dollar zusammensetzte, lag das Gros der Zuwendungen an $M c$ Cain über dieser Marke. Analog den Kommunikationsstrukturen insgesamt diente dem Bewerber der Demokraten hierbei das Internet als erweiterte Plattform: Für das Sammeln von finanziellen Mitteln wurden mit der Grassroots-Taktik möglichst viele Unterstützer über das Web aktiviert und mit dieser - wenn auch oftmals geringen Spende psychologisch gebunden: Schließlich war jeder Spender nominell Teil der Bewegung. Eine echte Innovation oder gar „Revolution“ “33 war diese Vorgehensweise vom Ansatz her nicht. Der erste Kandidat, der das Internet gezielt und aus damaliger Perspektive relativ erfolgreich zum Sammeln von Spenden einsetzte, war ausgerechnet McCain 2000. Vier Jahre später versuchte der Demokratische Bewerber Howard Dean einen ganz ähnlichen Weg, war aber insgesamt wesentlich weniger effektiv als Obama 2008.

Neu war dagegen die Instrumentalisierung der vielzitierten Kleinspender durch deren gezielte Integration in die Kampagne. Während anderswo Großkapital und einflussreiche Einzelpersonen mit ihren Spenden Einfluss nehmen, stünden die unzähligen Kleinspender - so die medial gern lancierte Botschaft - für eine urdemokratische Basisbewegung, die sich ganz im Sinne des omnipräsenten Überwortes Change fundamental vom bisherigen Washingtoner Politikstil unterscheide. Dass Obama aber primär mithilfe des kleinen Mannes in Amerikas höchstes Regierungsamt gelangt war, gehört in den Bereich der Legendenbildung: Wie alle anderen vor ihm in jüngerer Vergangenheit gewählten Präsidenten profitierte der Senator signifikant von einem sorgsam gepflegten Netzwerk potenter Unterstützer, wobei unter den so genannten Fat Cats neben Industriellen oder Immobilienmagnaten

40 Vgl. James Griffin, Campaign Finance in the United States, in: AICGS Issuebrief 25/2008, Washington D.C.

41 So Joshua Green, The Amazing Money Machine, in: The Atlantic vom Juni 2008, S. 5.

42 Spendengelder aller Kandidaten und weitere Details: Federal Election Commission, 2008 Presidential Campaign Finance, http://www.fec.gov/DisclosureSearch/mapApp.do (Abruf am 31. Januar 2009).

43 James A. Barnes, Online Fundraising Revolution, in: National Journal vom 19. April 2008. 
nicht zuletzt Großbanken wie Goldman Sachs, JPMorgan oder Lehman Brothers zu den spendabelsten Geldgebern gehörten. Hinzu kamen - für das Gesamtbudget ebenfalls höchst bedeutsam - unzählige „Bundlers“, also Großspender, die durch die autarke Organisation von Unterstützergruppen jeweils „gebündelt" mindestens 50.000 US-Dollar weitergereicht hatten. Dennoch besetzten die Kleinspender neben dem mobilisierenden Moment und ihrem tatsächlichen Beitrag eine spezifische Rolle, die Großspender schon allein wegen der gesetzlich festgelegten Obergrenzen von Wahlkampfspenden nicht oder nur bedingt erfüllen konnten: Sie waren es, die aufgrund ihrer schier unerschöpflichen Masse für einen kontinuierlichen Geldfluss und damit für die konstant hohe Liquidität der gesamten Kampagne sorgten.

\section{Wahlergebnis}

An der Wahl vom 4. November 2008 beteiligten sich über 131 Millionen US-Bürger, 69.456.897 davon gaben Barack Obama ihre Stimme. ${ }^{44}$ Nie zuvor in der Geschichte des Landes hatte ein Präsident eine quantitativ breitere Wählerbasis. John McCain erreichte 59.934.814 Stimmen und damit nur gut eine halbe Million weniger als der 43. Präsident George W. Bush bei seiner Wiederwahl 2004. Dieses absolut betrachtet beeindruckende Niveau beider Protagonisten wird jedoch relativiert durch drei Faktoren: demographische Entwicklung, Proportionalität und Wahlsystem.

Erstens gaben bei einer geringfügig von 60,1 auf 61,7 Prozent gestiegenen Wahlbeteiligung $^{45} 2008$ über neun Millionen Menschen mehr ihre Stimme ab als beim letzten Mal absolut eine Steigerung um rund sieben Prozent. Bei den Gesamtstimmen konnte George W. Bush zuletzt ebenfalls dank wachsender Bevölkerungszahlen einen entsprechenden Höchstwert erzielen, obwohl er proportional ein im Vergleich mit den meisten seiner Amtsvorgänger schwaches Ergebnis zu verbuchen hatte; im Jahr 2000 wurde der Kandidat mit den landesweit meisten Stimmen, Al Gore, bekanntermaßen nicht einmal Präsident. Betrachtet man zweitens die Verhältnismäßigkeit, lag Barack Obama bei den absoluten Stimmen mit gut sieben Prozentpunkten Differenz zwar klar vor seinem Kontrahenten. Historische Dimensionen wurden damit aber nicht erreicht: Mit Dwight D. Eisenhower, Lyndon B. Johnson, Ronald Reagan sowie George Bush Senior hatten gleich vier Kandidaten bei ihrer erstmaligen Wahl zum Präsidenten im Nachkriegsamerika einen proportional größeren Vorsprung. Dennoch erzielte Obama mit 52,92 Prozent (vgl. Tabelle 3) das beste Resultat eines Demokratischen Kandidaten seit Johnson. McCain kam auf 45,66 Prozent. Kandidaten anderer Parteien spielten praktisch keine Rolle. Und drittens sind die absoluten Stimmen - jedenfalls in ihrer landesweiten Gesamtbetrachtung - eine analytisch zwar aufschlussreiche, prozessual letztendlich jedoch irrelevante Größe: Um Präsident zu werden, ist einzig die Mehrheit im Wahlmännerkollegium entscheidend und somit der Gewinn der

44 Quelle und weiterführende Informationen auf der Website der Federal Election Commission, 2008 Official Presidential General Election Results, veröffentlicht am 22. Januar 2009, www.fec. gov/pubrec/fe2008/2008presgeresults.pdf (Abruf am 31. Januar 2009).

45 Anteil an der wahlberechtigten Bevölkerung; Quelle und weiterführende Informationen auf der Website des United States Election Projects, http://elections.gmu.edu/voter_turnout.htm (Abruf am 10. März 2009). 
meisten Wahlmännerstimmen, die in fast allen Bundesstaaten ${ }^{46}$ über das Prinzip „The Winner Takes It All“ ausschließlich dem jeweils siegreichen Kandidaten zufallen.

\begin{tabular}{|c|c|c|c|c|c|c|c|}
\hline Bundesstaat & $\mathrm{EV}^{*}$ & Obama & McCain & Bundesstaat & $\mathrm{EV}^{*}$ & Obama & McCain \\
\hline Alabama & 9 & 38,74 & 60,32 & Montana & 3 & 47,17 & 49,43 \\
\hline Alaska & 3 & 37,89 & 59,42 & Nebraska & 4 & 41,60 & 56,53 \\
\hline Arizona & 10 & 44,91 & 53,39 & Nevada & 5 & 55,15 & 42,65 \\
\hline Arkansas & 6 & 38,86 & 58,72 & New Hampshire & 4 & 54,13 & 44,52 \\
\hline California & 55 & 60,94 & 36,91 & New Jersey & 15 & 57,14 & 41,61 \\
\hline Colorado & 9 & 53,66 & 44,71 & New Mexico & 5 & 56,91 & 41,78 \\
\hline Connecticut & 7 & 60,59 & 38,22 & New York & 31 & 62,88 & 36,03 \\
\hline Delaware & 3 & 61,91 & 36,93 & North Carolina & 15 & 49,70 & 49,38 \\
\hline D.C. & 3 & 92,46 & 6,53 & North Dakota & 3 & 44,47 & 53,07 \\
\hline Florida & 27 & 50,91 & 48,10 & Ohio & 20 & 51,39 & 46,80 \\
\hline Georgia & 15 & 46,90 & 52,10 & Oklahoma & 7 & 34,35 & 65,65 \\
\hline Hawaii & 4 & 71,85 & 26,58 & Oregon & 7 & 56,75 & 40,40 \\
\hline Idaho & 4 & 35,96 & 61,30 & Pennsylvania & 21 & 54,48 & 44,17 \\
\hline Illinois & 21 & 61,88 & 36,76 & Rhode Island & 4 & 62,86 & 35,06 \\
\hline Indiana & 11 & 49,91 & 48,88 & South Carolina & 8 & 44,90 & 53,87 \\
\hline Iowa & 7 & 53,93 & 44,39 & South Dakota & 3 & 44,75 & 53,16 \\
\hline Kansas & 6 & 41,57 & 56,50 & Tennessee & 11 & 41,79 & 56,85 \\
\hline Kentucky & 8 & 41,15 & 57,37 & Texas & 34 & 43,64 & 55,39 \\
\hline Louisiana & 9 & 39,93 & 58,56 & Utah & 5 & 34,27 & 62,34 \\
\hline Maine & 4 & 57,71 & 40,38 & Vermont & 3 & 67,46 & 30,45 \\
\hline Maryland & 10 & 61,92 & 36,47 & Virginia & 13 & 52,63 & 46,33 \\
\hline Massachusetts & 12 & 61,80 & 35,99 & Washington & 11 & 57,35 & 40,27 \\
\hline Michigan & 17 & 57,35 & 40,90 & West Virginia & 5 & 42,57 & 55,68 \\
\hline Minnesota & 10 & 54,06 & 43,82 & Wisconsin & 10 & 56,22 & 42,31 \\
\hline Mississippi & 6 & 43,00 & 56,18 & Wyoming & 3 & 32,54 & 64,78 \\
\hline Missouri & 11 & 49,25 & 49,39 & Gesamt & 538 & 52,92 & 45,66 \\
\hline
\end{tabular}

46 Ausnahmen sind die Bundesstaaten Nebraska und Maine. Erstmals überhaupt kam es dabei tatsächlich zu einem Split der Stimmen in Nebraska, da die Stimmen aus den drei Kongresswahlbezirken dem auf dieser Ebene jeweils erfolgreichen Kandidaten zufallen. 
Von den insgesamt 538 Wahlmännerstimmen ${ }^{47}$ konnte Obama 365 auf sich vereinen, 173 gingen an McCain - ein komfortabler Vorsprung von 192 Stimmen und ein signifikant besseres Ergebnis als bei Amtsvorgänger Bush, der 2004 nur 35 Stimmen und 2000 lediglich fünf Wahlmänner mehr als sein jeweiliger Gegenkandidat aufbringen konnte. Der letzte Demokratische Präsident, Bill Clinton, lag dagegen 1992202 Stimmen und bei seiner Wiederwahl 1996 insgesamt 220 Stimmen vor seinem jeweiligen Opponenten. Die Reichweite des klaren Erfolges von Obama zeigt sich beim Blick auf einzelne Bundesstaaten ${ }^{48}$ : 28 von 50 Bundesstaaten sowie Washington D.C. konnte er für sich entscheiden, darunter alle Bundesstaaten, die 2004 auch der Bewerber der Demokraten John Kerry gewonnen hatte. Wie erwartet, gab es Siege in allen Demokratischen Hochburgen wie etwa sämtlichen Neuengland- und Pazifikstaaten, in Obamas „Heimatstaaten“ Illinois und Hawaii sowie den bevölkerungsreichen, traditionell eher Demokratisch orientierten Staaten New York, Pennsylvania, Michigan, Wisconsin und Minnesota. Umgekehrt war McCain wenig überraschend in den meisten Südstaaten, dem von evangelikalem Protestantismus geprägten Bible-Belt sowie partiell im Mittleren Westen erfolgreich. Um bei dieser Ausgangslage überhaupt Chancen auf den Gesamtsieg zu haben, hätte $M c$ Cain sämtliche „Battleground States" gewinnen und darüber hinaus den Demokraten noch den einen oder anderen Staat abnehmen müssen. Letztendlich erfolgreich waren die Republikaner in diesen 2008 besonders umkämpften Staaten aber nur in Montana, Arizona und Missouri. Alle anderen konnte Obama für sich entscheiden, darunter die bevölkerungsreichen und traditionell hart umkämpften Bundesstaaten Ohio und Florida, die bei den letzten beiden Wahlen mit den Ausschlag über Sieg und Niederlage gegeben hatten. Insgesamt war es den Demokraten in neun Bundesstaaten gelungen, die zuletzt Republikanische Mehrheit zu brechen. Besonders spektakuläre Verschiebungen gab es dabei unter anderem in Indiana und New Mexico; besonders symbolträchtig waren die Erfolge Obamas in North Carolina und Virginia, wo seit 1976 beziehungsweise seit 1964 kein Demokratischer Präsidentschaftskandidat mehr gewinnen konnte.

\section{Wahlentscheidung in demographischen und sozialen Gruppen}

\subsection{Alter}

Neben der Kandidatenorientierung und den Themen - sowie den in diesem Zusammenhang den Protagonisten zugeschriebenen Images beziehungsweise Kompetenzen ${ }^{49}$ - übernehmen im Abstimmungsverhalten auch demographische Größen und Sozialstruktur eine zentrale Funktion. ${ }^{50}$ Das wohl charakteristischste Teilergebnis der Wahl Obamas zum 44.

47 Die Anzahl der Wahlmännerstimmen eines jeden Bundesstaates entspricht der Zahl der Abgeordneten im Kongress. Zu diesen 535 Stimmen kommen drei Wahlmännerstimmen aus Washington D.C. Vgl. Kara Z. Buckley, Parties and Elections in America. The Electoral Process, Lanham 2004.

48 Überblick unter http://www.zdf.de/ZDFmediathek/content/390706 (Abruf am 6. November 2008).

49 Vgl. insgesamt Kapitel 2.1. und 2.2.

50 Allgemein dazu Angus Campbell / Philip E. Converse / Warren E. Miller / Donald E. Stokes, The American Voter, New York 1960. 
Präsidenten ist sein großer Erfolg bei jungen Amerikanern: Während der 47-jährige Jurist landesweit 53 Prozent erreichte, kam er bei den unter 30-jährigen Wählern auf 66 Prozent (vgl. Tabelle 4). Auch wenn die Demokratische Partei in diesem Alterssegment zuletzt bei den meisten Wahlen überproportional erfolgreich abschneiden konnte und die Zahl der psychologisch-integrativ an die Demokraten gebundenen ${ }^{51}$ Jungwähler in den letzten acht Jahren um fast zehn Prozentpunkte gewachsen ist, waren die Unterschiede zwischen Gesamtheit und der jüngeren Generation seit Vorliegen entsprechender Daten niemals zuvor größer. ${ }^{52}$ Bei den 30- bis 44-Jährigen lag Obama noch sechs Prozentpunkte vor McCain, bei den 45- bis 64-Jährigen - der quantitativ größten Wählergruppe - kam es zum Patt. Bei den ab 65-Jährigen lag McCain mit acht Prozentpunkten dagegen klar vor Obama. Diese maximalen Differenzen von 21 Punkten bei der altersspezifischen Analyse verweisen somit einerseits auf eine gestiegene Heterogenität der Wählerstrukturen beider Lager. 2004 lagen zwischen dem besten und dem schlechtesten Einzelergebnis in den Altersgruppen bei John Kerry nur acht Punkte Differenz, bei George W. Bush neun Punkte. Andererseits hat sich damit das altersspezifische Gefälle auf beiden Seiten verschärft: Während die Republikaner parallel mit dem Alter der Wähler immer mehr Unterstützung bekommen, nimmt die Zustimmung zu den Demokraten mit den Jahren kontinuierlich ab.

Wie relevant ein entsprechend starkes Abschneiden bei den unter 30-Jährigen für den Gesamtsieg Obamas war, zeigen die praktisch unveränderten Gruppenanteile an der Gesamtwählerschaft: Nach 17 Prozent im Jahr 2004 stellten die unter 30-Jährigen jetzt 18 Prozent aller Wähler. Besonders aufschlussreich ist in diesem Kontext die Kombination der Faktoren Alter und Hautfarbe, denn exakt diejenigen ethnischen Gruppen mit weit überdurchschnittlicher Zustimmung zu Obama waren bei den unter 30-jährigen Wählern am Wahltag klar überrepräsentiert: Während am 4. November 200813 Prozent aller Wähler Afro-Amerikaner waren, lag der entsprechende Anteil bei den unter 30-Jährigen bei 18 Prozent. Hispanics, insgesamt nur acht Prozent aller Wähler, waren in dieser jüngsten Wählergruppe zu 14 Prozent vertreten. Einen insgesamt ähnlichen Trend gibt es bei den Erstwählern: Diese stellten genau wie vor vier Jahren ein zwar unverändertes Kontingent von elf Prozent an der Gesamtwählerschaft, gaben aber zu 68 Prozent Obama und zu 31 Prozent $M c$ Cain ihre Stimme, wobei auch hier wieder überproportional viele Afro-Amerikaner und Hispanics erstmals überhaupt von ihrem Wahlrecht Gebrauch machten. Zuletzt votierten dagegen nur 53 Prozent der Erstwähler für John Kerry; George W. Bush kam in dieser Gruppe bei seiner Wiederwahl auf 46 Prozent.

\subsection{Geschlecht und Hautfarbe}

Eine weitere substanzielle Stütze für Obamas Wahlsieg waren die Frauen: 49 Prozent der Wähler, aber 56 Prozent der Wählerinnen stimmten für den Demokraten. McCain kam bei Männern auf 48 Prozent und bei Frauen auf 43 Prozent. Dabei ist die Tendenz von mehr

51 Zum Modell der Party Identification, vgl. Angus Campbell / Gerald Gurin / Warren E. Miller, The Voter Decides, Evanston 1954.

52 Altersgruppenspezifische Daten auf Basis von Exit Polls gibt es für US-Präsidentschaftswahlen seit 1972. Vgl. auch Scott Keeter / Juliana Horowitz / Alec Tyson, Young Voters in the 2008 Election, in: Pew Research Center Publications vom 12. November 2008. 


\begin{tabular}{|c|c|c|c|}
\hline & Gesamtanteile & Obama-Wähler & McCain-Wähler \\
\hline \multicolumn{4}{|l|}{ Alter } \\
\hline 18 bis 29 Jahre & 18 & 66 & 32 \\
\hline 30 bis 44 Jahre & 29 & 52 & 46 \\
\hline 45 bis 64 Jahre & 37 & 49 & 49 \\
\hline über 65 Jahre & 16 & 45 & 53 \\
\hline \multicolumn{4}{|l|}{ Hautfarbe } \\
\hline Weiße & 74 & 43 & 55 \\
\hline Schwarze & 13 & 95 & 4 \\
\hline Hispanics/Latinos & 8 & 66 & 32 \\
\hline \multicolumn{4}{|l|}{ Geschlecht } \\
\hline Männer & 47 & 49 & 48 \\
\hline Frauen & 53 & 56 & 43 \\
\hline \multicolumn{4}{|l|}{ Familieneinkommen } \\
\hline bis 50.000 US-\$ & 38 & 60 & 38 \\
\hline bis 100.000 US-\$ & 36 & 49 & 49 \\
\hline über 100.000 US- $\$$ & 26 & 49 & 50 \\
\hline \multicolumn{4}{|l|}{ Konfession / Religionszugehörigkeit } \\
\hline Protestanten/andere Christen & 54 & 45 & 54 \\
\hline Katholiken & 27 & 54 & 45 \\
\hline Juden & 2 & 78 & 21 \\
\hline Andere & 6 & 73 & 22 \\
\hline Konfessionslose & 11 & 75 & 23 \\
\hline \multicolumn{4}{|l|}{ Kirchoangshäufigkeit } \\
\hline wöchentlich & 40 & 43 & 55 \\
\hline gelegentlich & 42 & 57 & 42 \\
\hline nie & 16 & 67 & 30 \\
\hline \multicolumn{4}{|l|}{ formales Bildungsniveau } \\
\hline ohne Abschluss & 4 & 63 & 35 \\
\hline High School & 20 & 52 & 46 \\
\hline Some College / Associate Degree & 31 & 51 & 47 \\
\hline College & 28 & 49 & 48 \\
\hline postgraduiert & 17 & 58 & 40 \\
\hline
\end{tabular}

weiblicher Unterstützung zugunsten des Demokratischen Kandidaten ein altbekanntes Phänomen, das bei der letzten Präsidentschaftswahl 2004 proportional ähnlich stark und im Jahr 2000 sogar noch deutlicher aufgetreten war. Ebenfalls erwartungsgemäß war selbst in dieser Intensität - das Abschneiden Obamas mit 95 Prozent bei schwarzen Wählern, einer traditionell Demokratischen Kernklientel. Bei weißen Amerikanern, die nach 77 Prozent 2004 auch jetzt wieder mit 74 Prozent die weitaus stärkste und gemessen an ihrem tatsächlichen Bevölkerungsanteil weiter klar überrepräsentierte Wählergruppe stellten, lag Obama mit 43 Prozent deutlich hinter McCain mit 55 Prozent. Dabei betrug der Vorsprung McCains bei weißen Männern 16 Prozentpunkte und bei weißen Frauen noch immerhin sieben Punkte. Schon eher überraschend war - was Veränderungen bei den ethnischen Gruppen betrifft - die starke „Rückkehr“ der Hispanics beziehungsweise Latinos: Nachdem die Republikaner bei der letzten Präsidentschaftswahl in dieser Bevölkerungs- 
gruppe noch ein Plus von neun Prozentpunkten verzeichnen konnten, stand bei dieser Wahl ein Minus von zwölf Punkten. Letztendlich gaben zwei Drittel der Hispanics Obama und nur 31 Prozent McCain ihre Stimme. An der Urne bleiben die Hispanics aber eine relative Minderheit: Inzwischen wird gut jeder sechste Amerikaner dieser ethnischen Gruppe zugerechnet $^{53}$, ihr Anteil an der Gesamtwählerschaft lag dagegen bei lediglich acht Prozent. Obwohl sie die am schnellsten wachsende Bevölkerungsgruppe sind, hat sich ihr Gesamtgewicht im US-Elektorat seit der letzten Präsidentschaftswahl damit nicht geändert.

\subsection{Konfession und Kirchgang}

Das konfessionelle Wahlverhalten der US-Amerikaner war bei der Wahl des 44. Präsidenten in seinen Grundzügen prinzipiell stabil, die Stärken und Schwächen der beiden großen Lager blieben weitgehend erhalten. Analog der Gesamtheit haben sich jedoch die Gewichte in praktisch allen konfessionellen Subgruppen zugunsten des Demokratischen Kandidaten verschoben. So ist die besonders wertkonservative und mit einem Viertel aller Wähler besonders starke Strömung der Evangelikalen beziehungsweise „Wiedergeborenen Christen“ weiter eine Domäne der Republikaner, wo McCain - nur marginal schlechter als Bush vier Jahre zuvor - 74 Prozent der Stimmen holte. Unter allen regelmäßig praktizierenden Protestanten erreichte $M c$ Cain bei durchschnittlichen Einbußen 66 Prozent und unter allen regelmäßigen Kirchgängern noch 55 Prozent. Bei den Katholiken, im Wahlverhalten zuletzt noch gespalten und nach den Protestanten beziehungsweise anderen Christen mit anteilig 27 Prozent die zweitgrößte konfessionelle Wählergruppe, gewann Obama nun eine Mehrheit von 54 Prozent. Die stärksten Veränderungen gab es aber unter den konfessionslosen Wählern, die allerdings nur elf Prozent der Wähler am 4. November 2008 ausmachten: In dieser Gruppe fiel McCain acht Prozentpunkte hinter das Ergebnis von Bush aus dem Jahr 2004 zurück, die Demokraten sind hier umgekehrt traditionell stark und erzielten mit 75 Prozent ein besonders gutes Resultat.

\subsection{Bildung und Einkommen}

Bei der bildungsspezifischen Analyse gibt es - anders als etwa beim Alter - keinen gleichförmigen Trend. Barack Obama war mit 63 Prozent bei den formal niedriger gebildeten Wählern ohne Schulabschluss besonders erfolgreich, jedoch auch bei postgraduierten Hochschulabsolventen mit 58 Prozent. Ein relatives Defizit gibt es gemessen am Gesamtergebnis dagegen bei graduierten College-Absolventen, wo Obama und McCain etwa gleich stark abschnitten. Bei US-Bürgern mit formal mittlerer Bildung lag Obama vier Prozentpunkte und bei Wählern mit High School als höchstem Schulabschluss sechs Punkte vor seinem Kontrahenten.

Besonderen Zuspruch erhielt Obama von Amerikanern mit geringen finanziellen Ressourcen, die an der Wahlurne allerdings etwas weniger stark präsent waren als vier Jahre zuvor. 75 Prozent der Wähler mit weniger als 15.000 US-Dollar Jahreseinkommen, 60 Prozent

53 United States Census Bureau, American Community Survey 2007, Hispanic or Latino Origin by Race, http://factfinder.census.gov/home/en/official_estimates_2007.html (Abruf am 19. März 2009). 


\begin{tabular}{|l|c|c|c|c|}
\hline Tabelle 5: Einstellungen und Parteiidentifikation \\
\hline & $\begin{array}{c}2004 \\
\text { alle }\end{array}$ & $\begin{array}{c}2008 \\
\text { alle }\end{array}$ & $\begin{array}{c}2008 \\
\text { Obama-Wähler }\end{array}$ & $\begin{array}{c}2008 \\
\text { McCain-Wähler }\end{array}$ \\
\hline Einstellungen & & & & \\
konservativ & 33 & 34 & 20 & 78 \\
moderat & 45 & 44 & 60 & 39 \\
liberal & 22 & 22 & 88 & 10 \\
\hline Parteiidentifikation & 36 & 32 & 9 & 89 \\
Republikaner & 38 & 39 & 89 & 10 \\
Demokraten & 26 & 29 & 52 & 44 \\
Unabhängige & & & \\
\hline Quelle: Edison Media Research / Mitofsky International, a.a.O. (Fn. 27); Edison Media Research / Mi- \\
tofsky International, a.a.O. (Fn. 28).
\end{tabular}

derjenigen mit bis zu 30.000 und noch 55 Prozent derjenigen mit bis zu 50.000 US-Dollar im Jahr gaben Obama ihre Stimme. In den darüber liegenden Einkommensgruppen bis 200.000 US-Dollar ist bei den Präferenzen eine starke Polarisierung zu konstatieren, was für das Demokratische Lager im Vergleich zu 2004 zumindest einen Teilerfolg bedeutet. Die weitaus stärkste Bewegung gab es jedoch bei Jahreseinkommen jenseits der 200.000 USDollar: Hatte Bush bei seiner Wiederwahl vor vier Jahren hier noch ein äußerst komfortables Polster von 17 Prozentpunkten, schafften die Demokraten mit Obama jetzt selbst in dieser ökonomisch besonders potenten Wählergruppe einen Vorsprung von sechs Punkten.

\subsection{Parteiorientierungen und Einstellungen}

Bei den grundsätzlichen Parteiorientierungen gibt es kurz-, aber auch mittelfristig viel Konstanz, die politisch-ideologischen Grundhaltungen sind praktisch unverändert (vgl. Tabelle 5). Dabei hat Obama innerhalb der unverändert großen Demokratischen Kernklientel, also derjenigen Wähler mit entsprechender Parteibindung, nicht besser abschneiden können als Kerry im Jahr 2004. Allerdings war er sowohl bei den parteipolitisch unabhängigen als auch bei den Republikanisch orientierten Wählern sichtbar erfolgreicher, wobei die unabhängigen Wähler 2008 einen etwas größeren, die Republikaner mit Parteibindungen einen etwas kleineren Anteil stellten als zuvor.

Der übergreifende Erfolg Obamas zeigt sich auch entlang ideologischer Konfliktlinien: Während sich weiterhin eine relative Mehrheit der Amerikaner politisch in der Mitte verortet und daneben weiterhin deutlich mehr konservative als liberale Wähler an die Urne gehen, ist es ihm gelungen, in jeder dieser drei Grundströmungen erfolgreicher zu rekrutieren als Kerry vier Jahre zuvor. Davon unabhängig haben insgesamt doppelt so viele ehemalige Wähler Bushs jetzt für Obama gestimmt als es ehemalige Kerry-Wähler gab, die nun für McCain votierten. Hinzu kommt, dass auch die zuletzt weit verbreitete „Negativwahl“ massiv abgenommen hat: Hatte 2004 fast jeder dritte Wähler seine Entscheidung auch damit begründet, dass so der Kandidat der Gegenseite nicht zum Zug käme, hat sich dieser Anteil 2008 fast halbiert. ${ }^{54}$

54 Vgl. Pew Research Center, Some Final Thoughts on Campaign '08, in: Pew Research Center Publications vom 8. Dezember 2008. 


\section{Kommt nach dem Sieg der Wandel?}

Die US-Präsidentschaftswahl vom 4. November 2008 markiert eine doppelte Zäsur: Symbolisch ist die Wahl Barack Obamas zum 44. US-Präsidenten rund anderthalb Jahrhunderte nach Abschaffung der Sklaverei und knapp ein halbes Jahrhundert nach der formellen Beendigung der Rassensegregation weit mehr als ein Zeichen, vor allem auch deshalb, weil die Hautfarbe bei der Entscheidung praktisch keine Rolle spielte. Perspektivisch mindestens ebenso wichtig scheint die politische Botschaft: Das Votum der US-Bürger steht nicht nur für den Wunsch nach einem administrativen Wechsel, sondern nach einem umfassenden politischen Neubeginn. Nach acht langen Jahren einer massiv polarisierenden Bush-Regierung, zu deren Beginn ein nationales Trauma stand, dem eine simple Unterscheidung der Welt in Gut und Böse, verlustreiche Kriege sowie ein erheblicher Ansehens- und Bedeutungsverlust der Supermacht auf internationalem Parkett folgten, kanalisierte Barack Obama diesen Wunsch am besten. Strategisch brillant, mit einer nahezu perfekten Kampagne und begünstigt von Kontextbedingungen hat er seinen Wählern einen radikalen Kurswechsel versprochen. Zugleich appellierte Obama an uramerikanische Tugenden und weckte mit einfachen Slogans wie „Yes, we can“ neben Zustimmung an der Urne in der Bevölkerung Hoffnung und politische Begeisterung. Das Elektorat ist hierbei in seinen Grundkoordinaten stabil geblieben, denn die Stärken und Schwächen der politischen Lager reflektieren auch bei dieser Wahl klassische Muster des US-amerikanischen Wahlverhaltens. Auch wenn die Gewichte zugunsten der Demokraten neu justiert wurden, war der Wechsel ohne ein umfassendes sozialstrukturelles Realignment möglich.

Ganz ähnlich wie bei der Wiederwahl Bushs im Jahr $2004^{55}$ resultiert der Sieg Obamas weniger aus signifikanten strukturellen Veränderungen in einzelnen gesellschaftlichen, sozialen oder demographischen Gruppen, sondern basiert auf gewachsener Unterstützung in den meisten Bevölkerungsteilen im gesamten Land. In 46 von 50 Bundesstaaten konnten die Demokraten ihr Ergebnis verbessern, in 28 davon sowie in der Hauptstadt sind sie nun stärkste Partei, im Senat und Repräsentantenhaus besitzen sie eine Mehrheit. Doch nach dem großen Sieg warten jetzt noch größere Aufgaben: Während ein euphorisches Europa auf einen transatlantischen Neubeginn hofft und Obamas Administration tatsächlich sehr schnell neue Töne anschlägt, sind die USA de facto außenpolitisch weiter in Kriege und diverse Konflikte verstrickt. Innenpolitisch befindet sich das Land in der schwersten Krise seit Jahrzehnten. Neben den zahlreichen sozialen und gesellschaftlichen Problemen bricht die US-Wirtschaft dramatisch ein, die Arbeitslosenzahlen steigen rapide, und Experten malen das Gespenst einer tiefen Rezession. Ob und wie der neue US-Präsident diese Herausforderungen meistern kann, bleibt abzuwarten. Dass ein beeindruckender Wahlsieg und ein Regierungswechsel hierfür höchstens einen Anfang bedeuten, ist Barack Obama bewusst: "This victory alone is not the change we seek. "56

55 Vgl. Bernhard Kornelius, Die Mär von der Moral. Eine genaue Analyse zeigt, dass der US-Präsident den Wahlsieg nicht nur religiösen und konservativen Gruppen verdankt, in: SZ vom 14. Dezember 2004.

56 So Barack Obama in seiner Victory Speech während der Wahlnacht vom 4. November 2008 in Chicago, Illinois. Als Video unter anderem unter http://elections.nytimes.com/2008/results/president/speeches/obama-victory-speech.html (Abruf am 10. März 2009). 